\title{
Direct Numerical Simulation of Twin Swirling Flow Jets: Effect of Vortex-Vortex Interaction on Turbulence Modification
}

\author{
Wenkai Xu, ${ }^{1}$ Nan Gui, ${ }^{1}$ Liang Ge, ${ }^{1}$ and Jie Yan $^{2}$ \\ ${ }^{1}$ College of Mechanical and Transportation Engineering, China University of Petroleum, Beijing 102249, China \\ ${ }^{2}$ China Academy of Space Technology, Beijing 10094, China
}

Correspondence should be addressed to Nan Gui; zjuguinan@zju.edu.cn

Received 14 April 2014; Accepted 21 June 2014; Published 9 July 2014

Academic Editor: Fu-Yun Zhao

Copyright (C) 2014 Wenkai Xu et al. This is an open access article distributed under the Creative Commons Attribution License, which permits unrestricted use, distribution, and reproduction in any medium, provided the original work is properly cited.

A direct numerical simulation (DNS) was carried out to study twin swirling jets which are issued from two parallel nozzles at a Reynolds number of $\operatorname{Re}=5000$ and three swirl levels of $S=0.68,1.08$, and 1.42, respectively. The basic structures of vortex-vortex interaction and temporal evolution are illustrated. The characteristics of axial variation of turbulent fluctuation velocities, in both the near and far field, in comparison to a single swirling jet, are shown to explore the effects of vortex-vortex interaction on turbulence modifications. Moreover, the second order turbulent fluctuations are also shown, by which the modification of turbulence associated with the coherent or correlated turbulent fluctuation and turbulent kinetic energy transport characteristics are clearly indicated. It is found that the twin swirling flow has a fairly strong localized vortex-vortex interaction between a pair of inversely rotated vortices. The location and strength of interaction depend on swirl level greatly. The modification of vortex takes place by transforming largescale vortices into complex small ones, whereas the modulation of turbulent kinetic energy is continuously augmented by strong vortex modification.

\section{Introduction}

Swirling flows exist in many engineering applications, such as gas turbine combustors, internal combustion engines, cyclone separators, and industrial burners. It is proven as an effective mixing enhancement approach. Swirling flows are extensively studied in many aspects, such as vortex breakdown [1], instability [2], and recirculation zones [3]. Theoretical and experimental approaches are two important tools to research swirling flow. Most early theories were developed to characterize the conditions of vortex breakdown and predict its location and criterion. Recent theoretical studies tried to describe the whole process and predict the internal structure. Although lots of theories have been proposed, none of them agreed well with all features of breakdown [4]. Therefore, many experiments were conducted to investigate swirling flows in detail.

For example, Zohir [5] studied heat transfer characteristics and pressure drop for turbulent airflow with propeller swirl generator. It indicated that inserting the propeller downstream provides considerable improvement of heat transfer rate higher than inserting the propeller upstream. Xie et al. [6] investigated the effect of rotational acceleration on flow and heat transfer in swirl microchannels. They showed complicated flow and heat transfer characteristics at different acceleration directions, and the acceleration effects are reduced in swirl microchannels. Iyogun et al. [7] used PIV approach to investigate the stability of swirling nonpremixed flame with different nozzle geometries. Adzlan and Gotoda [8] studied the behavior of stable vortex breakdown occurring in a coaxial swirling jet with a density difference experimentally and considered the effect of density difference on the height of stagnation point. Litvinov et al. [9] performed an experimental study of strongly swirling air flow with high Reynolds number to characterize precessing vortex core (PVC) and proved an analytical model of a helical vortex which is useful to describe or predict PVC.

With development of computational technology and improvement of computational speed, investigators applied various numerical methods to study swirl flow and gained lots of satisfied results. For example, Younis et al. [10] presented an objective model for the press-strain correlation to predict 
momentum and scalar transport in turbulent swirling flows. They assessed the performance of their model by comparing to experimental data of weakly and strongly swirling jets and obtained close agreement between the two. Moreover, large eddy simulation (LES) and direct numerical simulation (DNS) were widely used to numerically study swirling flow. For example, Roux et al. [11] studied turbulent flow within a complex swirl combustor with LES and experiments for both cold and reacting flows and found that the comparison between mean LES results and experimental data of velocity fields was satisfactory. Therefore, their results confirm the remarkable predictive capacity of LES methods and also highlight the need for well-defined boundary conditions. Müller and Kleiser [12] investigated vortex breakdown in a compressible swirling jet flow by LES using the approximate deconvolution model; their simulation showed good agreement with experimental results. Strong unsteady structure, PVC, and coherent structure of nonreactive swirl flow were captured in LES study of Yang and Kær [13]. LES was also utilized to investigate many other aspects, including complex swirl flows [14], external intermittency [15], and modeling swirl flows with hybrid LES/RANS model [16]. Moreover, Patil and Tafti [14] implemented LES to simulate complex high Reynolds number swirl flows, where the wall model and the synthetic eddy method were utilized to reduce computational resource. Additionally, DNS results of fluid flow and mixing of a recirculating swirling flow at moderate Reynolds number were compared to experimental data [17]. An overall good agreement between DNS and experiment was shown. Freitag and Klein [18] simulated a recirculating, swirling flow at a Reynolds number of 5000 using DNS. Excellent agreement was shown between the detailed one and two-point statistics of numerical results and experimental data.

However, previous researches almost focus on singular swirling flow or singular swirling flow jet. Only few studies contributed to the investigation of multiple swirling flows or twin jets [19-21]. As a result, to better understand the characteristics of twin swirling flow, the aim of this paper is to study the twin swirling jets and investigate interaction between the twin swirling jet flows by DNS method.

\section{Numerical Description}

2.1. Governing Equations. Single-phase swirling flows without chemical reaction and distinct heat transfer were simulated. Temperature is not changed and the variation of density can be ignored. To implement direct numerical simulation of swirling flows, the three-dimensional, time-dependent, incompressible, viscous Navier-Stokes equations are solved in Cartesian coordinates:

$$
\begin{gathered}
\frac{\partial u_{i}}{\partial x_{i}}=0 \\
\frac{\partial u_{i}}{\partial t}=-\frac{\partial u_{i} u_{j}}{\partial x_{j}}-\frac{1}{\rho} \frac{\partial p}{\partial x_{i}}+\frac{\partial}{\partial x_{j}} \nu\left(\frac{\partial u_{i}}{\partial x_{j}}+\frac{\partial u_{j}}{\partial x_{i}}\right),
\end{gathered}
$$

where $\rho$ is constant fluid density and $\nu$ is kinematic viscosity. The governing equations are solved by the finite difference
TABLE 1: Parameters used in the numerical simulation.

\begin{tabular}{lc}
\hline Characteristic diameter $d(\mathrm{~mm})$ & 0.1 \\
Scales of the flow domain & $20 d \times 10 d \times 10 d$ \\
Grid numbers, $N_{x} \times N_{y} \times N_{z}$ & $512 \times 256 \times 256$ \\
Reynolds number, Re & 5000 \\
Swirl number, $S$ & $0.68 ; 1.08 ; 1.42$ \\
Mean inlet axial velocity $U_{0}(\mathrm{~m} / \mathrm{s})$ & 1.52 \\
Fluid density $\left(\mathrm{kg} / \mathrm{m}^{3}\right)$ & 1000 \\
Fluid dynamic viscosity $(\mathrm{Pa} \cdot \mathrm{s})$ & 25 \\
Time step, $\Delta t(\mu \mathrm{s})$ & 2.5 \\
Total simulation time, $T_{s}$ & 50 \\
\hline
\end{tabular}

method. The convection term is discretized by the upwind compact schemes [22]. Space derivatives and pressuregradient terms are discretized by the sixth order compact difference schemes [23]. The third order explicit schemes are used to deal with the boundary points, to maintain the global fourth order spatial accuracy. The fourth order Runge-Kutta schemes [24] are used for time integration. The pressurePoisson equation is solved to obtain the pressure by using the fourth order finite difference method [25].

The direct numerical simulation of single swirling jet has been carried out in one of my previous works [26] and the numerical method has been validated through the comparison with experimental results. Thus, the same numerical method is used in this work. The configuration of flow is sketch in Figure 1 (the left inset). In the previous work, jet diameter $d$ is $0.4 \mathrm{~mm}$, and the flow domain dimensions are $30 d \times 10 d \times 10 d$. The grids number is $384 \times 128 \times 128$ and the Reynolds number is 606. However, in the present study, $d=0.1 \mathrm{~mm}$ is used, and the domain dimensions are $20 d \times 10 d \times 10 d$. The grid numbers are set $512 \times 256 \times 256$ and Reynolds number is 5000. To guarantee requirement of spatial resolution for direct numerical simulation, grid numbers must be greater than a threshold value of $N_{g}=$ $N_{x 0} N_{y 0} N_{z 0}$, which is proportional to $\mathrm{Re}^{9 / 4}$. It is known that the grid number in any dimension, for instance, $N_{x 0}$, is proportional to $\mathrm{Re}^{3 / 4}$, if the computational shape is similar. Moreover, the spatial spacing scale $\Delta S$ is proportional to $1 / N_{x 0}$, which means that $\Delta S$ is proportional to $1 / \mathrm{Re}^{3 / 4}$. However, the ratio of $1 / \mathrm{Re}^{3 / 4}$ of this work to previous one is about $1: 4.76$, and the ratio of spatial spacing of this work to previous work is $1: 8$, which means that the spatial spacing in this work is smaller than the required threshold value and the grid is fine enough to maintain the spatial resolution for DNS. In simulation, we use dimensionless variables, and their corresponding real scales are listed in Table 1.

2.2. Boundary Conditions. The swirl is imparted through an initial distribution of tangential velocity at the inlet boundary the same as our preview work [26]. The swirl number is defined by the ratio of maximum tangential velocity to the mean axial velocity:

$$
S=\frac{2 \cdot \max \left\{U_{\theta}\right\}}{U_{0}},
$$



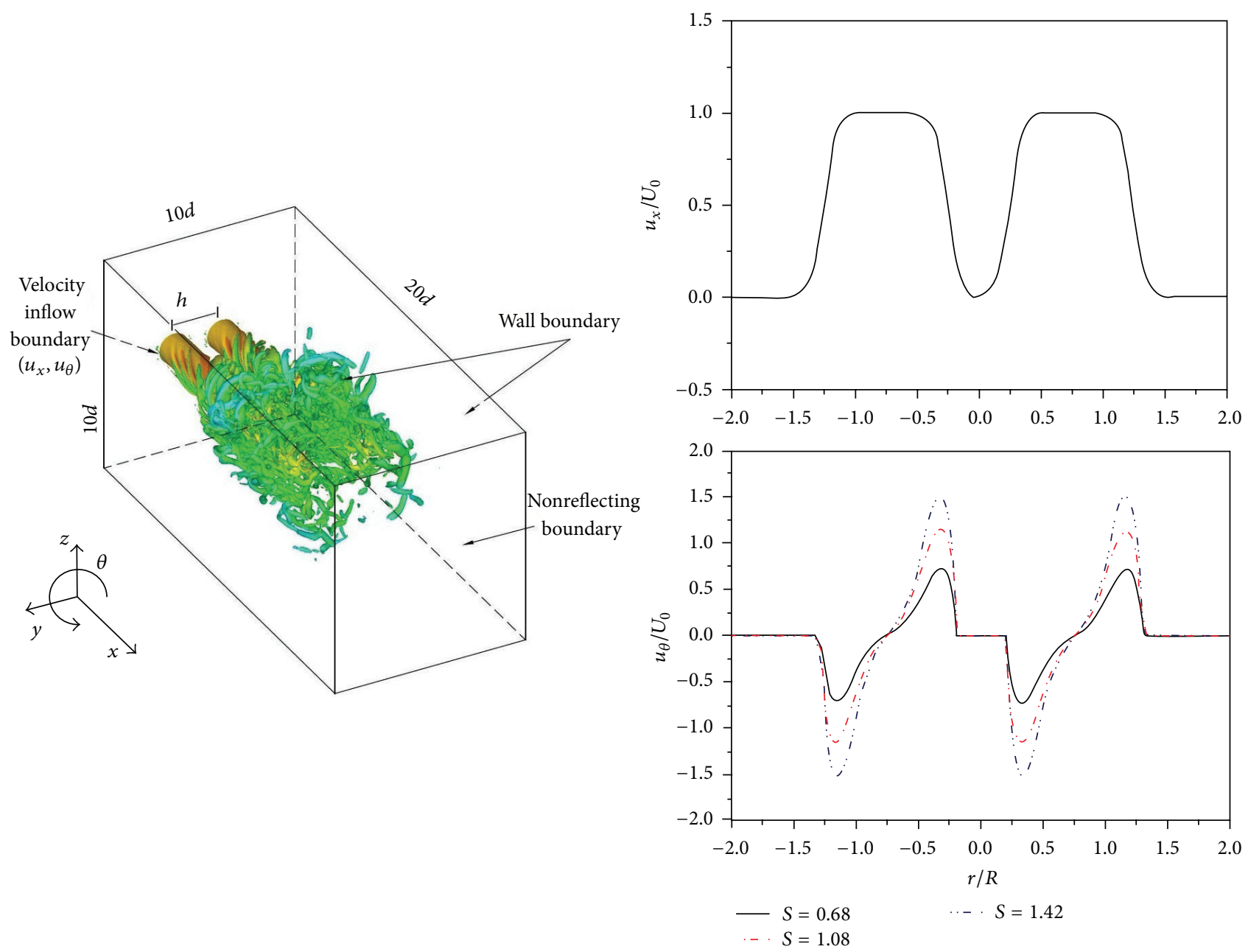

FIGURE 1: The flow configuration and inflow velocity profiles.

where $U_{\theta}$ is the tangential velocity and $U_{0}$ is the inlet velocity. For the inflow boundary, nozzles are mounted inside the flow domain. At the inlet of the nozzle, the particularly specified profiles of swirl and axial velocities are set as the inflow boundary condition (Figure 1, the right insets). The flow field characteristics change as the swirl number increases and the vortex breakdown takes places when the level of swirl exceeds a critical value (e.g., $S=1.42$ [26]). For this reason, we chose three typical values of swirl levels of $S=0.68,1.08$, and 1.42 for detailed study. Moreover, both the jets are of anticlockwise swirl.

With regard to the inflow fluctuation, it is set as zero [27]. Moreover, as the Reynolds number is kept at a relatively high value, the fluctuation is stronger than the previous simulation. In this way, the evolution behavior of vortices is due to the intrinsic nature of the swirling flow. At the end of the flow domain, a nonreflecting boundary condition is applied as the outflow boundary $[28,29]$. The other boundary conditions are set as Dirichlet conditions which are nonslip wall boundaries. Similar simulation conditions are also used in one of our previous studies [27].

\section{Results and Discussions}

The main object of present study is to explore the characteristics of flow field in twin swirling jets and examine the correlation between the twin swirling jet flows through comparison of flow variables, such as vortex evolution, mean velocity, and root mean square (r.m.s.) of velocity fluctuations.

3.1. Temporal Evolution of Vortex Structure. Twin swirling jets share some common characteristics with singular swirling jet. For instance, in swirling jet, vortex-breakdown takes place when the level of swirl is high enough. A view of temporal variation of vortex structures in the three-dimensional twin swirling jets in the lateral and spanwise plane is illustrated in Figure 2 from $t=2.5 \mathrm{~ms}$ to $10 \mathrm{~ms}$. For Figures 2(a), 2(b), and 2 (c), obvious vortex interactions between the twin jets under $S=0.68,1.08$, and 1.42 , respectively, are shown. The twin jets under a large swirl level of $S=1.42$ are interacting with each other at the very beginning (Figure $2(\mathrm{c}-\mathrm{i}), t=2.5 \mathrm{~ms}$ ), whereas they are almost independent with each other under 

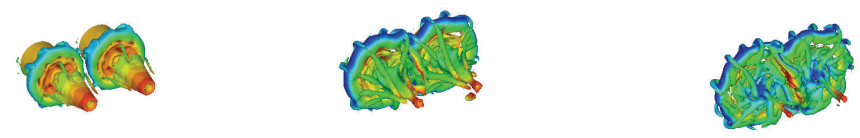

$a-i$

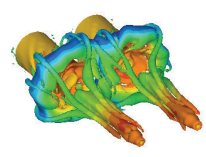

a-ii

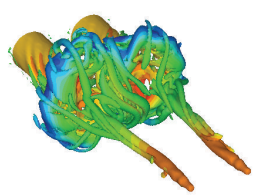

a-iii

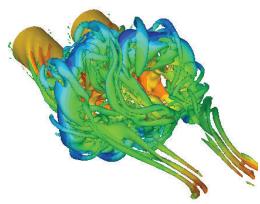

a-iv

(a) b-i

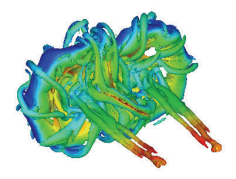

b-ii

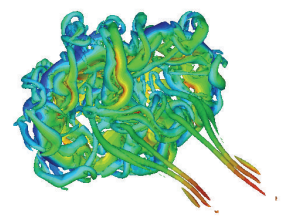

b-iii

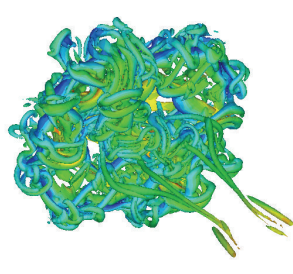

b-iv

(b)

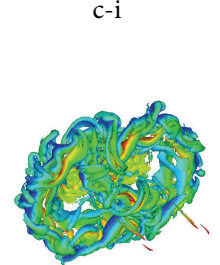

c-ii

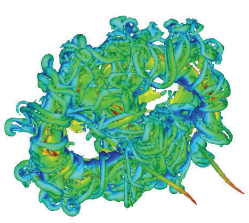

c-iii

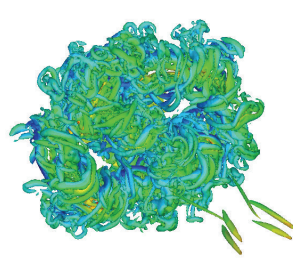

c-iv

(c)

Figure 2: Temporal variation of vortex structures in the three-dimensional twin swirling jets in $y$ - $z$ plane for $S=0.68$ (a), 1.08 (b), and 1.42 (c), respectively, from $t=2.5 \mathrm{~ms}$ to $10 \mathrm{~ms}$ (from i to iv correspondingly).

a low level (Figure 2(a-i)). Thereafter, the interaction of vortices between these twin jets becomes more and more evident and complex (from $t=5.0 \mathrm{~ms}$ to $t=10 \mathrm{~ms}$, Figures 2(a), 2(b), and 2(c)-ii to iv). At first, the vortices in the middle of the twin jets contact each other. Because these vortices are rotating in the opposite directions, the contact procedure makes the modification of a "ring"-shaped vortex to the fairly complex "S"-shaped (Figures 3(a) and 3(b)) large structure vortex. Then, more and more small-scale vortices are generated as the developments and interactions between the twin swirling jets are going on. The quantity of small-scale vortices is increased as the levels of swirl increase, as a larger swirl level could cause a more intensive interaction between the twin jets and therefore induce more small-scale vortices (Figures 2(a) to 2(c)).

It is also noticed that the "S"-shaped large structure of vortex can be interpreted by the contact procedure of the twin jets. As sketched in Figure 3(c), the twin jets are all rotating anticlockwise, forming two large rings of vortex rotating in the same direction. Simultaneously, the fluids are rotating about the large "ring"-shaped main structure of vortex, and there exists a contact point in the middle of the two rings, where rotating directions of fluids are opposite. As a result, there should be a fairly intensive interaction at the contact point, where the rotating motion is ideally assumed to be reduced to zero. However, this cannot always be accomplished and a series of small-scale induced vortices is generated after the contact point, which is named and indicated as postcontact regions in Figure 3(c). As a result, the " $\mathrm{S}$ "-shaped large structure of vortex distribution is established, and it is composed of a fairly large number of small-scale complex vortices.

3.2. Axial Variation of Turbulent Fluctuation in the Near Field. To illustrate the effect of vortex interaction between the twin jets, Figures 4, 5, and 6 illustrate the axial variation 


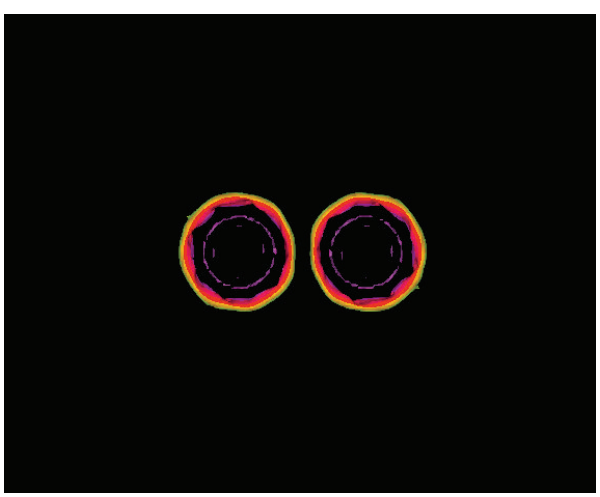

(a)

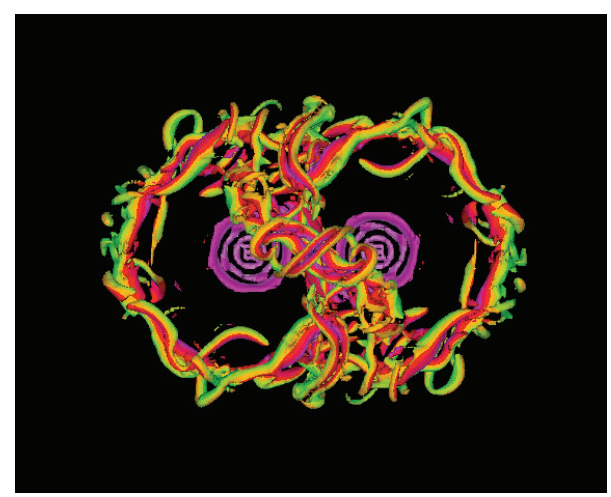

(b)

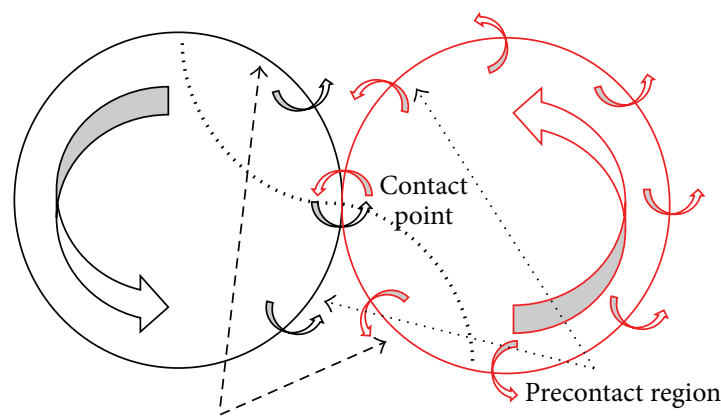

Postcontacting region

(c)

FIGURE 3: A "ring"-shape (a) and "S"-shape (b) vortex and a sketch of the inversely rotating large scale vortices (c).

of turbulent fluctuation velocities, such as root mean square values of velocity component $\left(u^{\prime}, v^{\prime}, w^{\prime}\right)$ in the $x, y$, and $z$ directions, at $S=0.68,1.08$, and 1.42 and in comparison to their counterparts of single swirling jet flow, respectively.

As shown in Figure 4(a) ( $u^{\prime}$ at $\left.S=0.68\right)$, it can be seen that the effect of vortex interaction on modification of axial variation of $u^{\prime}$ in twin jets becomes evident after $x=3 d$. After that, the $u^{\prime}$ in twins jets is larger than that in single jet. It shows that the vortex interaction between the twin jets can augment the axial turbulent fluctuation in twin jets. For $u^{\prime}$ at $S=1.08$ (Figure 4(b)), the interaction takes place after $x=2 d$, taking place earlier than $S=0.68$ for its a bit wider spread of vortex. The $u^{\prime}$ after $2 d$ is increased by vortex-vortex interaction slightly. Moreover, the diffusion of vortex around $x=2 d, 3 d$, and $4 d$ in twin jets is more intensive than that in single jet. Therefore, the secondary peak values of $u^{\prime}$ around $y=7-8 d$ disappear. With even more strongly swirling flows $(S=1.42$, Figure $4(c))$, the turbulent fluctuation of $u^{\prime}$ around the middle region of the twin jets is significantly augmented even in the nearest region of $x=1 d$. Thereafter, the axial variations of $u^{\prime}$ for twin jets and single jet are almost consistent with each other, although it is a bit larger in twin jets than in single jet. These results show that the interaction of vortices between the twin jets modifies, or more precisely augments, the turbulent feature of the jets, especially in the middle region. However, this type of turbulence modification in large swirl flows occurs within a fairly near field region around the nozzle outlet. After that, in the far field, the characteristics of turbulent fluctuation are almost the same, at least, not evidently modified.

Similar results can be observed for the fluctuation velocities of $v^{\prime}$ and $w^{\prime}$ in Figures 5 and 6, respectively. It is found that the interaction of vortex between the twin jets increases $v^{\prime}$ and $w^{\prime}$ after a short axial distance $(x \geq 3 d)$ in the low swirl jets $(S=0.68$, Figures $5(\mathrm{a})$ and $6(\mathrm{a}))$, especially in the peripheral region of the twin jets, whereas it deceases the turbulent fluctuation in the right middle of the jets slightly. In larger swirl twin flows $(S=1.08$, Figures $5(\mathrm{~b})$ and $6(\mathrm{~b})$ and $S=1.42$, Figures 5(c) and 6(c)), the interaction occurs earlier (around $x \geq 1 d$ ).

In Figure 5(b) for $v^{\prime}$ at $S=1.08$ around $x=1 d$, it is attenuated in the right middle region which is caused by the right contact of two inversely rotating vortices. After that, $v^{\prime}$ is larger in twin jets than in single jets. But the degree of increase is rapidly reduced due to the strong interaction and cannot be maintained in the downstream region. Around $x=4 d$, $v^{\prime}$ in twins jets is almost totally dissipated, whereas it has not been attenuated so greatly by the naturally diffusion motion of vortex in the single jet until it moves further downstream $(x \geq 5 d)$. For $w^{\prime}$ at $S=1.08$ in Figure 6(b), the trends are similar.

In Figure 5(c) for $S=1.42$, the augmentation of $v^{\prime}$ around $x=1 d$ is very clear, similar to that for $u^{\prime}$ in Figure $4(\mathrm{c})$. Around $x=2 d$, the two peaks of $v^{\prime}$ in single jet around $y=$ $8 d$ and $2.5 d$ are caused by the natural diffusion and spread motion of vortex. However, in twin jets, the augmentation 


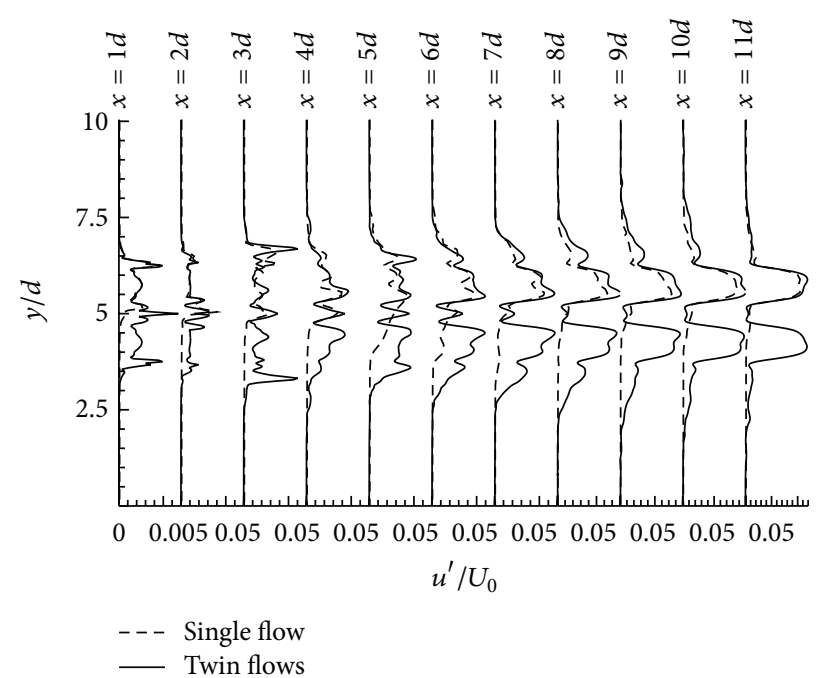

(a)

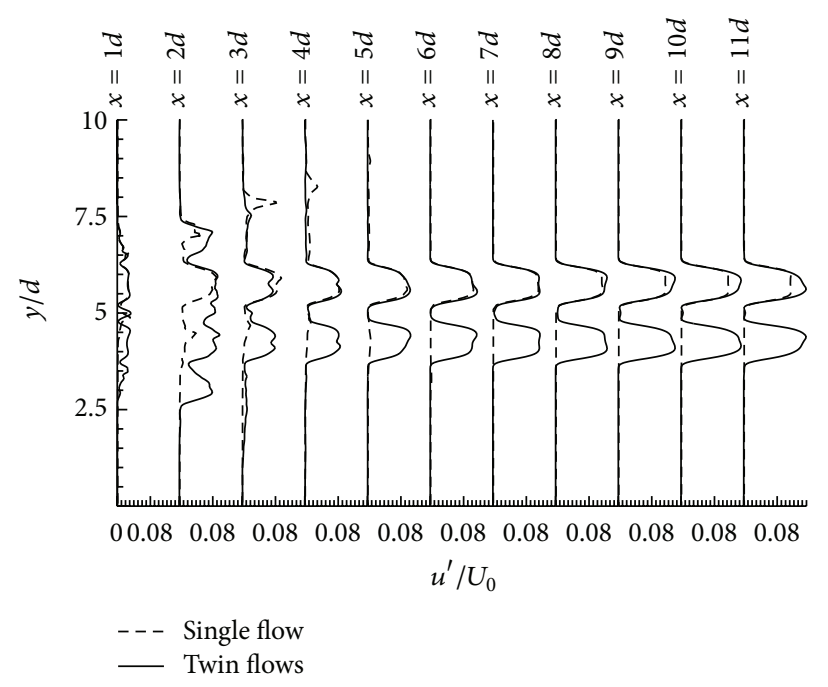

(b)

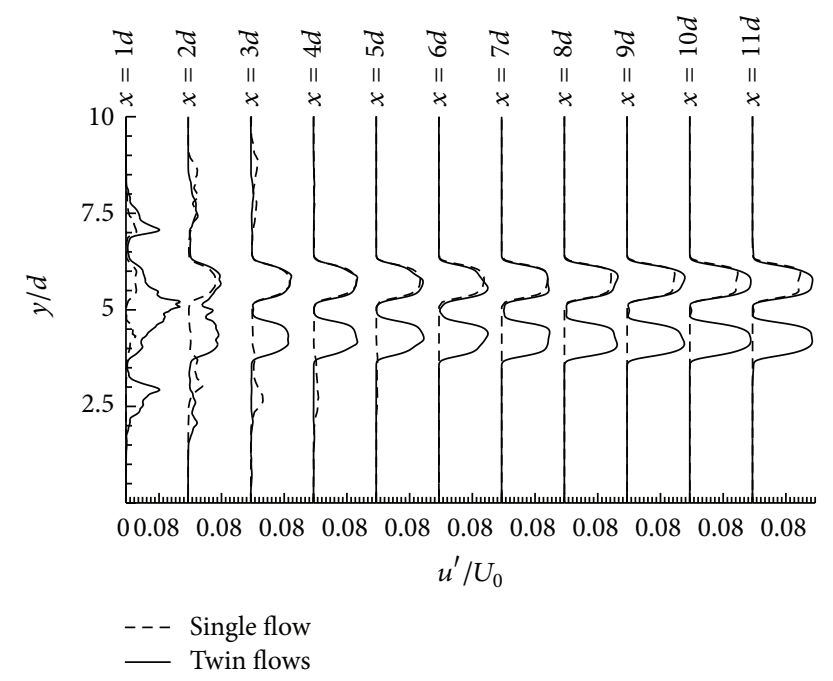

(c)

FIGURE 4: Axial variation of turbulent fluctuation velocity $u^{\prime}$ in the $x$ direction under $S=0.68$ (a), 1.08 (b), and 1.42 (c), respectively, in comparison to their counterparts of single swirling jet flows.

of $v^{\prime}$ in the middle region is still very large. Moreover, the spread motion and diffusion of vortex in $y$ direction are not as efficient as those in single jet, because the strong vortex interaction dissipates a lot of energies. Therefore, the fluctuations $v^{\prime}$ in twin jets after $x=2 d$ are almost completely reduced. The similar trends can be observed again for $w^{\prime}$ at $S=1.42$ in Figure 6(c).

3.3. Axial Variation of Turbulent Fluctuation in the Far Field. On the other hand, it can be clearly observed from Figure 4 to Figure 6 that turbulent fluctuation velocities $\left(u^{\prime}, v^{\prime}, w^{\prime}\right)$ after a certain distance look like a similar trend of variation, showing the possible characteristics of self-similarities. For example, for low swirl flows $(S=0.68)$, it occurs after about $x=10 d$, whereas it is about $x \geq 5 d$ for high swirl flows $(S=1.08$ and $S=1.42)$.
Thus, to validate the self-similar distribution of fluctuation velocity, Figures $7(\mathrm{a}), 7(\mathrm{~b})$, and $7(\mathrm{c})$ show the axial distributions of normalized turbulent fluctuation velocities for $S=0.68, S=1.08$, and $S=1.42$, respectively. The normalization procedure is done as follows, taking $u^{\prime}$ for example: (1) find the maximum value of $u^{\prime}$ in the $y$ /lateral direction; (2) compute the half of $\max \left(u^{\prime}\right)$; (3) calculate the lateral distance between the positions of $\max \left(u^{\prime}\right)$ and half $\max \left(u^{\prime}\right)$, named $y_{b} ;(4)$ divide the $u^{\prime}$ by $\max \left(u^{\prime}\right)$ and divide $y$ by $y_{b}$ to obtain the normalized $u^{\prime}$.

From Figure 7, it is clearly observed that the normalized $u^{\prime}$ follows an almost perfect consistent distribution, which validates the existence of self-similar turbulent fluctuation characteristics in the far field. Besides, it is seen from Figure 7 (a) that the self-similar trend of $u^{\prime}$ in low swirl flows is not established until $x=11 d$, before which an obvious 

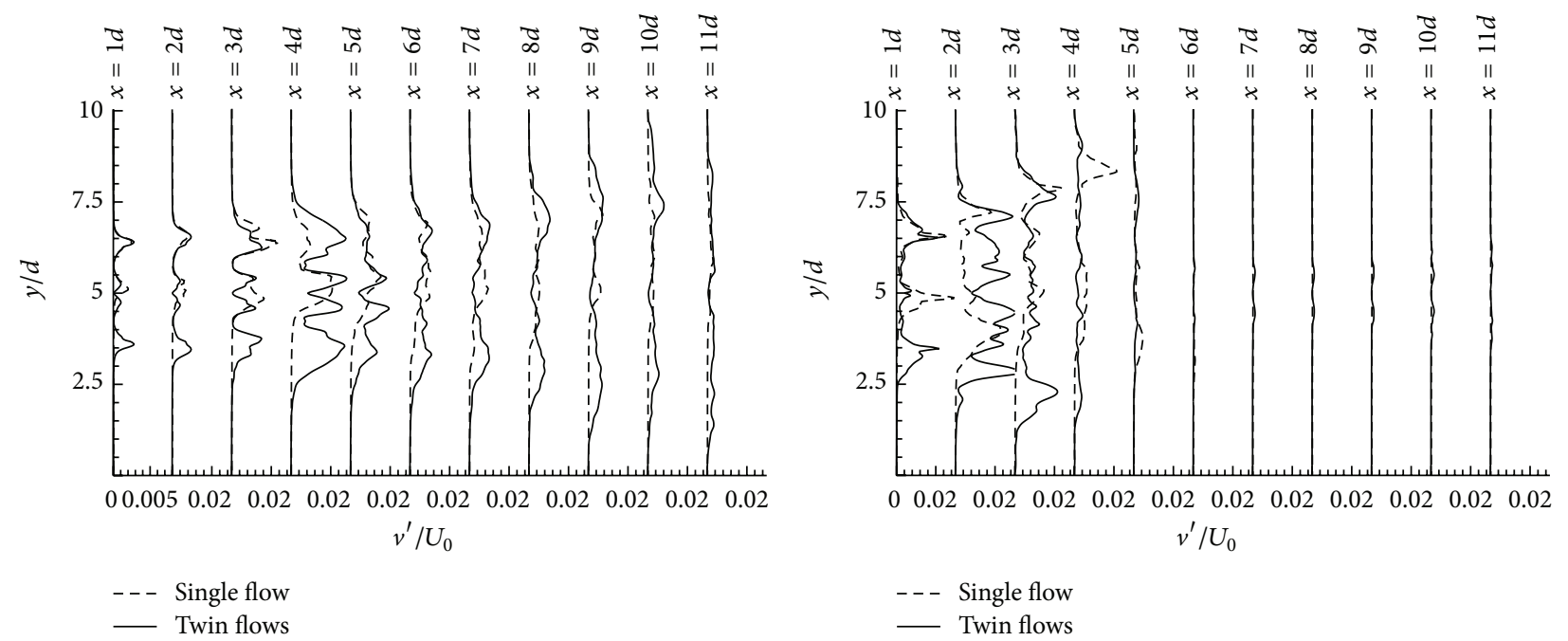

(a)

(b)

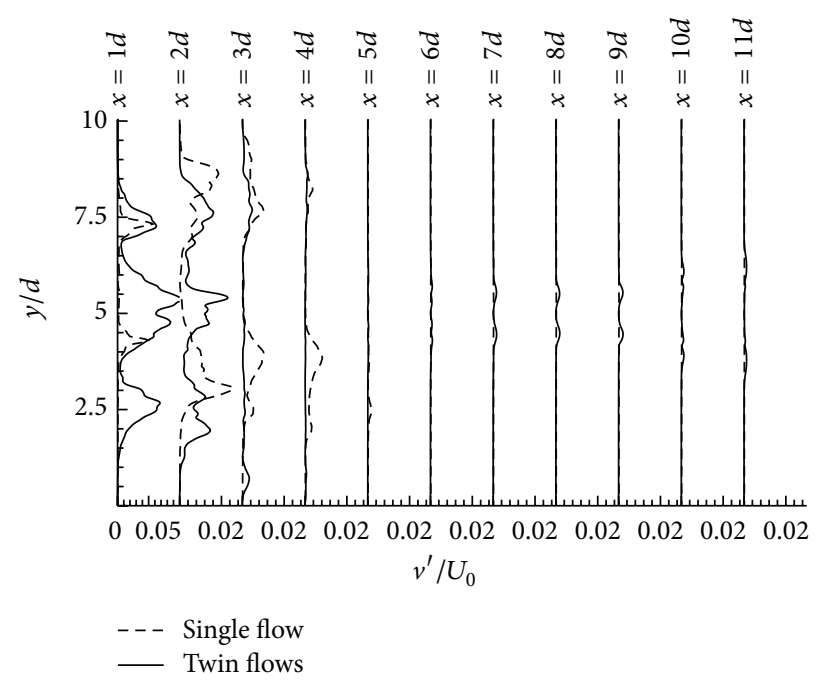

(c)

Figure 5: Axial variation of turbulent fluctuation velocity $v^{\prime}$ in the $y$ direction under $S=0.68$ (a), 1.08 (b), and 1.42 (c), respectively, in comparison to their counterparts of single swirling jet flows.

discrepancy of $u^{\prime}$ exists from the normalized uniform selfsimilar distribution.

In addition, recalling the feature of direct jet without swirl, the similarity is caused by the self-preserving characteristics of the jets. In direct jets, the turbulent kinetic energy is dissipated mainly by the vortex motion and diffusion in shear layer. However, in swirling jets or twin swirling jets, the turbulent kinetic energy can be reduced not only by shear but also by swirl motion. Thus, it should be dissipated more quickly than that in direct jets. For this reason, the selfsimilarity distribution takes place much earlier in high swirl jets than in low swirl jets, especially in twin swirling jets.

\subsection{Axial Variation of Second Order Velocity Fluctuations.} Turbulence characteristics can be deeply studied from the normalized secondorder fluctuation velocity profiles, such as $u^{\prime} v^{\prime}$ and $u^{\prime} w^{\prime}$ profiles, which illustrate detailed feature of correlation of turbulent velocity fluctuations. The aforementioned normalized first order fluctuation of axial velocities always has only one peak value and shares very similar shape. However, the curves of higher order correlated fluctuation velocities are relatively more complex than each component. They could change more rapidly with no explicit patterns and could be influenced by interaction between the twin swirling jets considerably too.

Distributions of higher order velocity fluctuations profiles of $u^{\prime} v^{\prime}$ and $u^{\prime} w^{\prime}$ for both singular swirling flow and twin swirling flows are illustrated in Figures 8 and 9, respectively. When the swirling level is low, the profiles of $u^{\prime} v^{\prime}$ and $u^{\prime} w^{\prime}$ have large amplitudes of peaks in the axial ranges from $x=2 d$ to $x=6 d$, while the amplitudes of peaks are much smaller in the far flow field (Figures 8(a) and 9(a)). However, when the swirl number increases to 1.08 , large variation of distribution of $u^{\prime} v^{\prime}$ and $u^{\prime} w^{\prime}$ in $y$ direction mainly occurs from $x=1 d$ to 

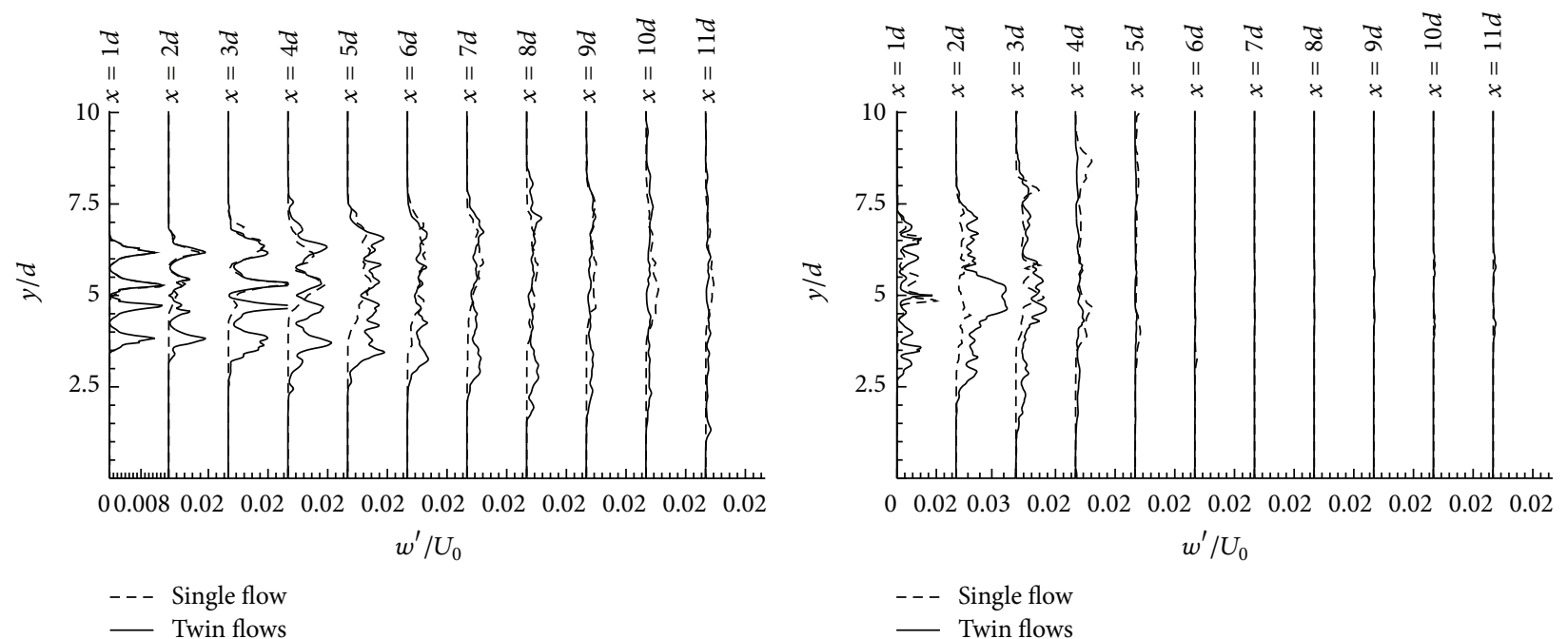

(a)

(b)

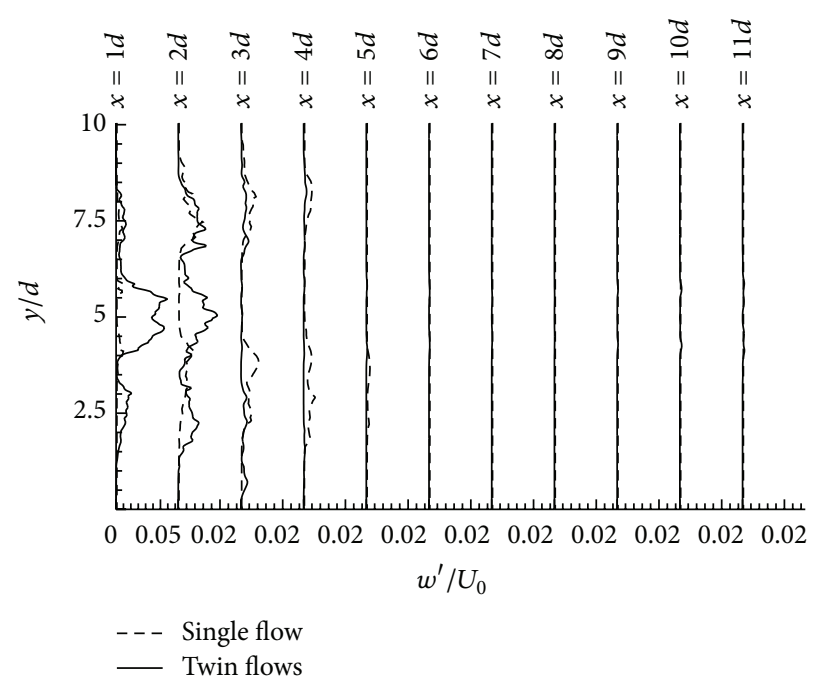

(c)

Figure 6: Axial variation of turbulent fluctuation velocity $w^{\prime}$ in the $z$ direction under $S=0.68$ (a), 1.08 (b), and 1.42 (c), respectively, in comparison to their counterparts of single swirling jet flows.

$4 d$. Their amplitudes are rapidly decayed after $x=5 d$ (Figures 8 (b) and 9(b)). When the swirl level increases to 1.42, obvious variations of $u^{\prime} v^{\prime}$ and $u^{\prime} w^{\prime}$ profiles only exist in the immediate near field of $x / d<3$, and their amplitudes are nearly zero in the downstream thereafter.

If the correlation between twin swirling flows is low and can be ignored, the $u^{\prime} v^{\prime}$ and $u^{\prime} w^{\prime}$ profiles in twin swirling jets should be mainly consistent with those in singular swirling jet. However, no matter what the swirl level is, obvious differences of $u^{\prime} v^{\prime}$ and $u^{\prime} w^{\prime}$ profiles between the twin swirling flows and the singular swirling flow exist in the region between the nozzles, although the difference is fine near the nozzle exit where the turbulent intensity is weak when the swirling level is low. The difference is increased with distance to nozzle exit as the turbulent intensity increases resulting from turbulence development, and it is decreased slightly after $x / d=5$.

In particular, quite distinguished differences around the axial locations, for example, $x / d=2$ for $S=1.08$ and $x / d=1$ for $S=1.42$ (Figures 8(b) and 9(b)), can be found. For moderate swirl level flows, the turbulence develops faster than the low level flows. Therefore, distinct differences appear at earlier locations even around $x / d=1$. However, the difference decays rapidly after $x / d=4$, which is caused by turbulence decay, especially in high swirl flows.

In addition, for $S=1.08$, the amplitudes of peaks of $u^{\prime} v^{\prime}$ and $u^{\prime} w^{\prime}$ profile in twin swirling flows are much larger than that of single swirling flow at $x / d=2$, whereas they are smaller in twins swirling flows than in single swirling flow at $x / d=3$. It is a consequence of turbulence overdissipation caused by the locally significantly augmented 


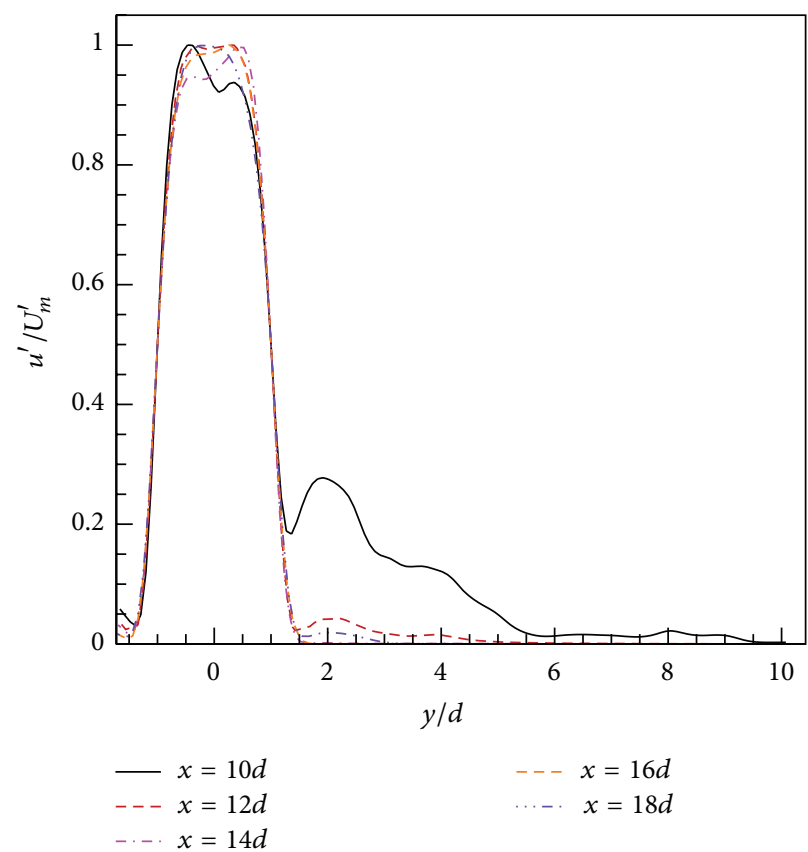

(a)

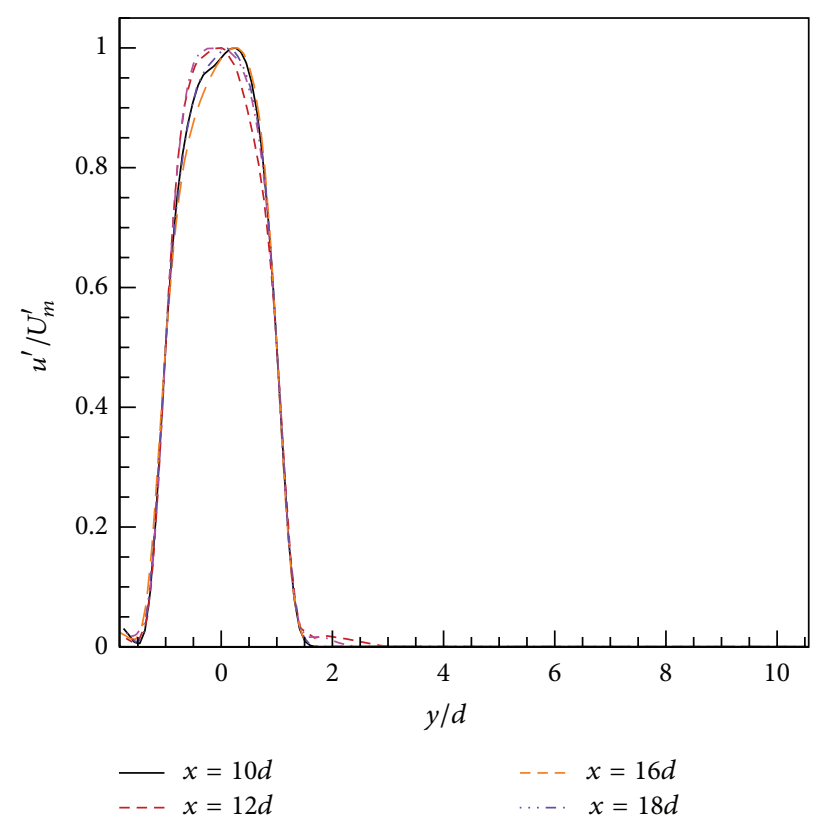

(b)

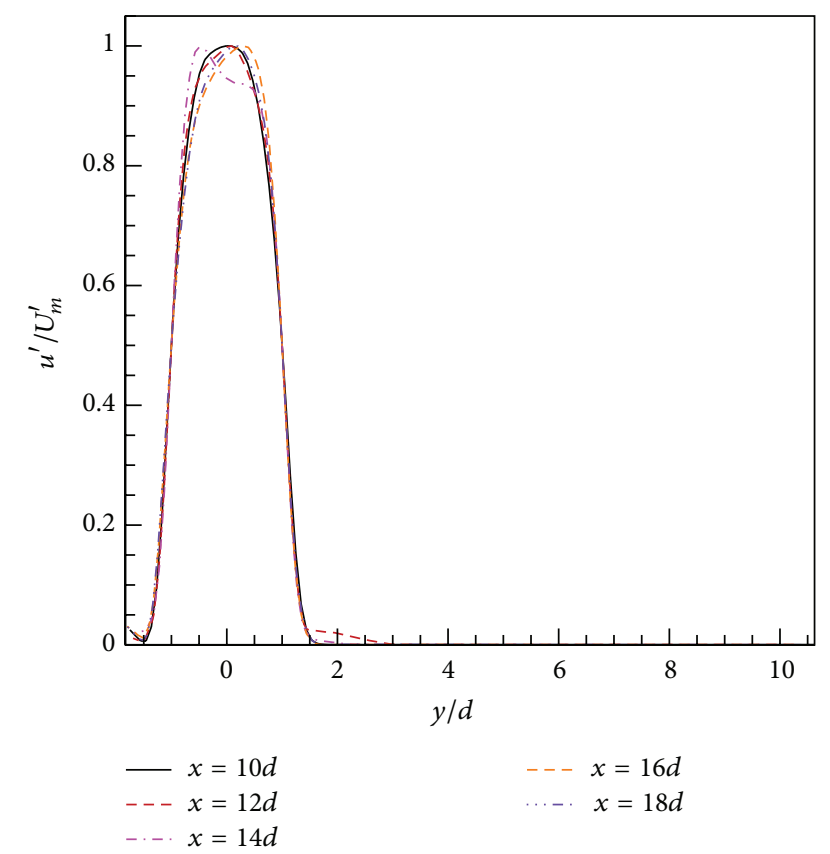

(c)

Figure 7: Axial distribution of normalized turbulent fluctuation velocity after $x=10 d$ for $S=0.68$ (a), $S=1.08$ (b), and $S=1.42$ (c), respectively.

vortex interaction around $x / d=2$. Similar phenomenon also occurs around $x / d=1$ and $x / d=2$ for $S=1.42$.

3.5. Axial Variation of Turbulent Kinetic Energy Associated with Reynolds Stress Tensor. As mentioned above, the distributions of higher order velocity fluctuation varying with the distance from nozzle exit are caused by the evolution of flow, which results in turbulent intensity modification. Therefore, the profiles of higher order velocity fluctuation can roughly illustrate turbulence evolution. In this section, we define the sum of squares of the second order velocity fluctuation (namely, the Reynolds stress tensor $u_{i}^{\prime} u_{j}^{\prime}$ ) on the central symmetrical plane in the $z$-direction to quantitatively demonstrate the streamwise variation of turbulence. Consider

$$
E_{i j}(x)=\int_{z=\text { center }}\left(u_{i}^{\prime} u_{j}^{\prime}\right)^{2} d y, \quad i, j, k=x, y, z .
$$




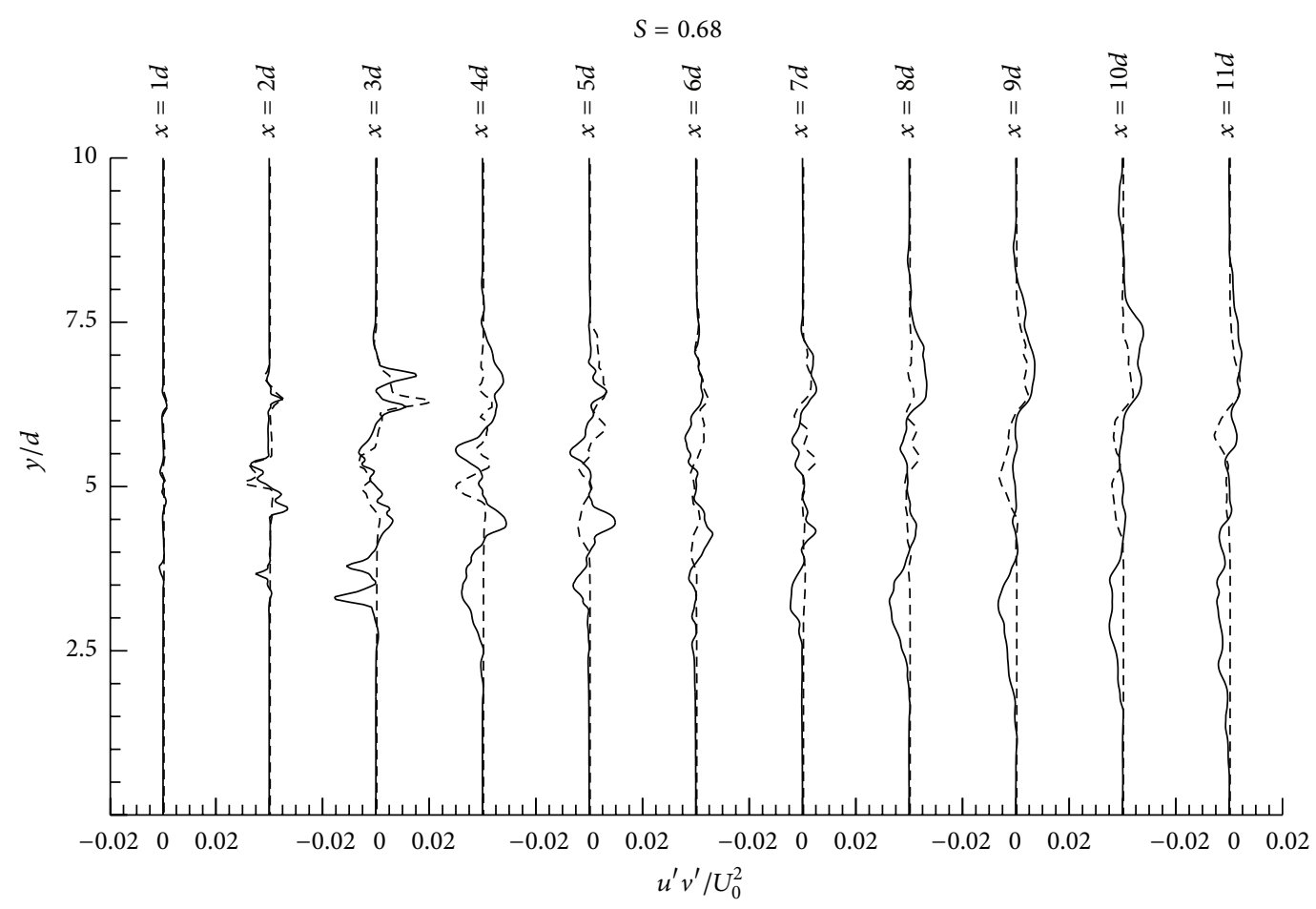

\section{- - - Single flow}

(a)
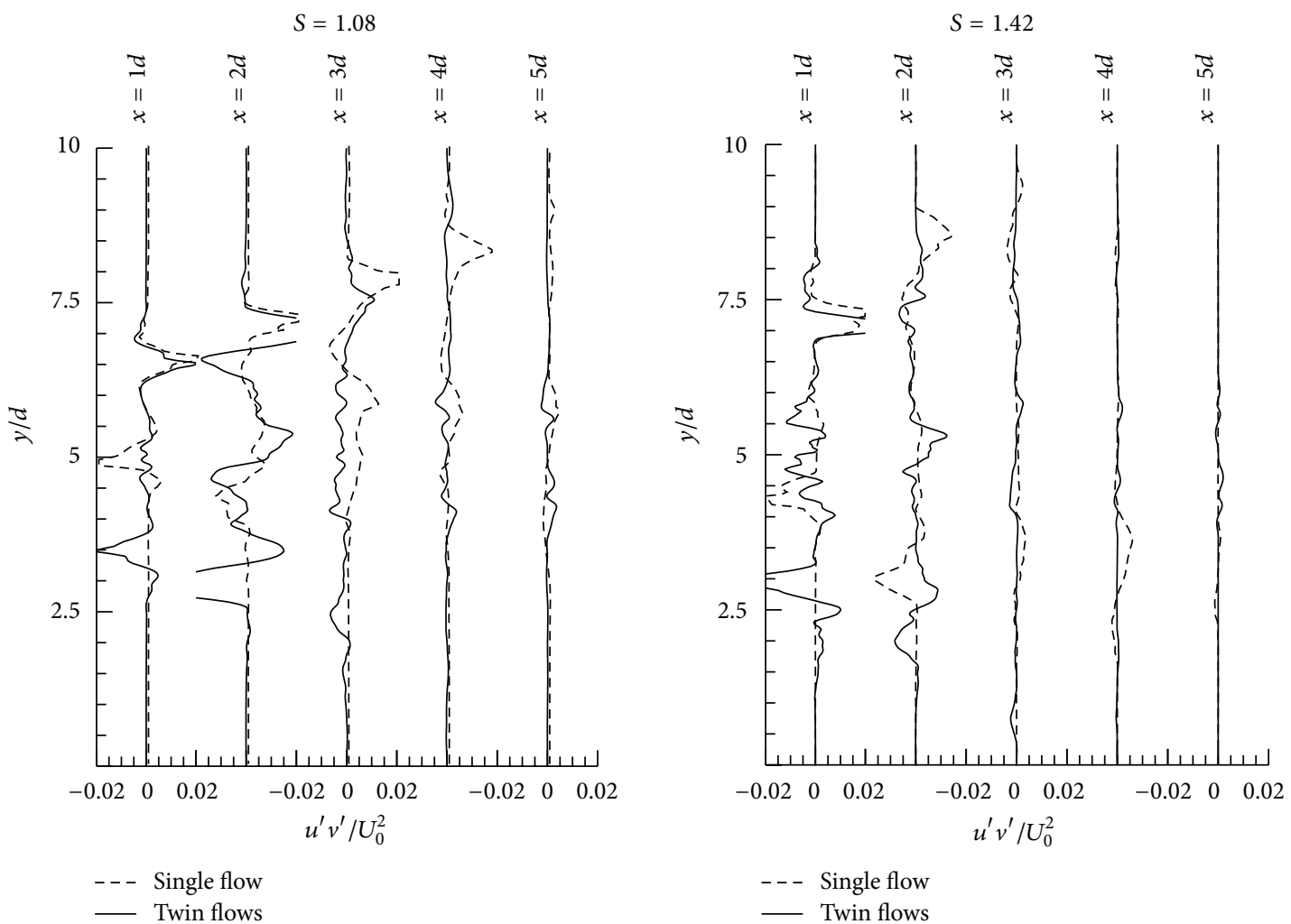

(b)

Figure 8: The second order velocity fluctuation profiles of $u^{\prime} v^{\prime}$ for $S=0.68$ (a) and $S=1.08$ and $S=1.42$ (b) in singular and twin swirling flows. 


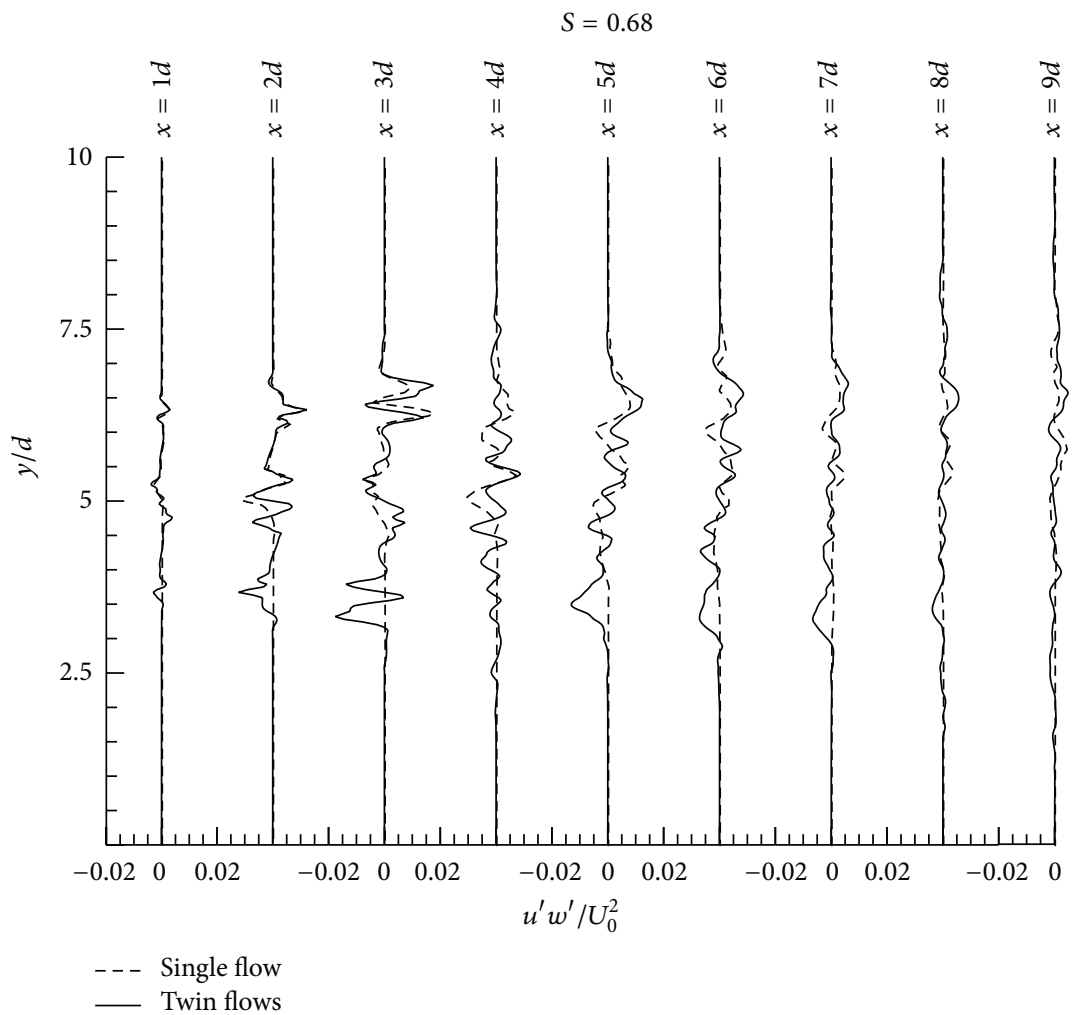

(a)
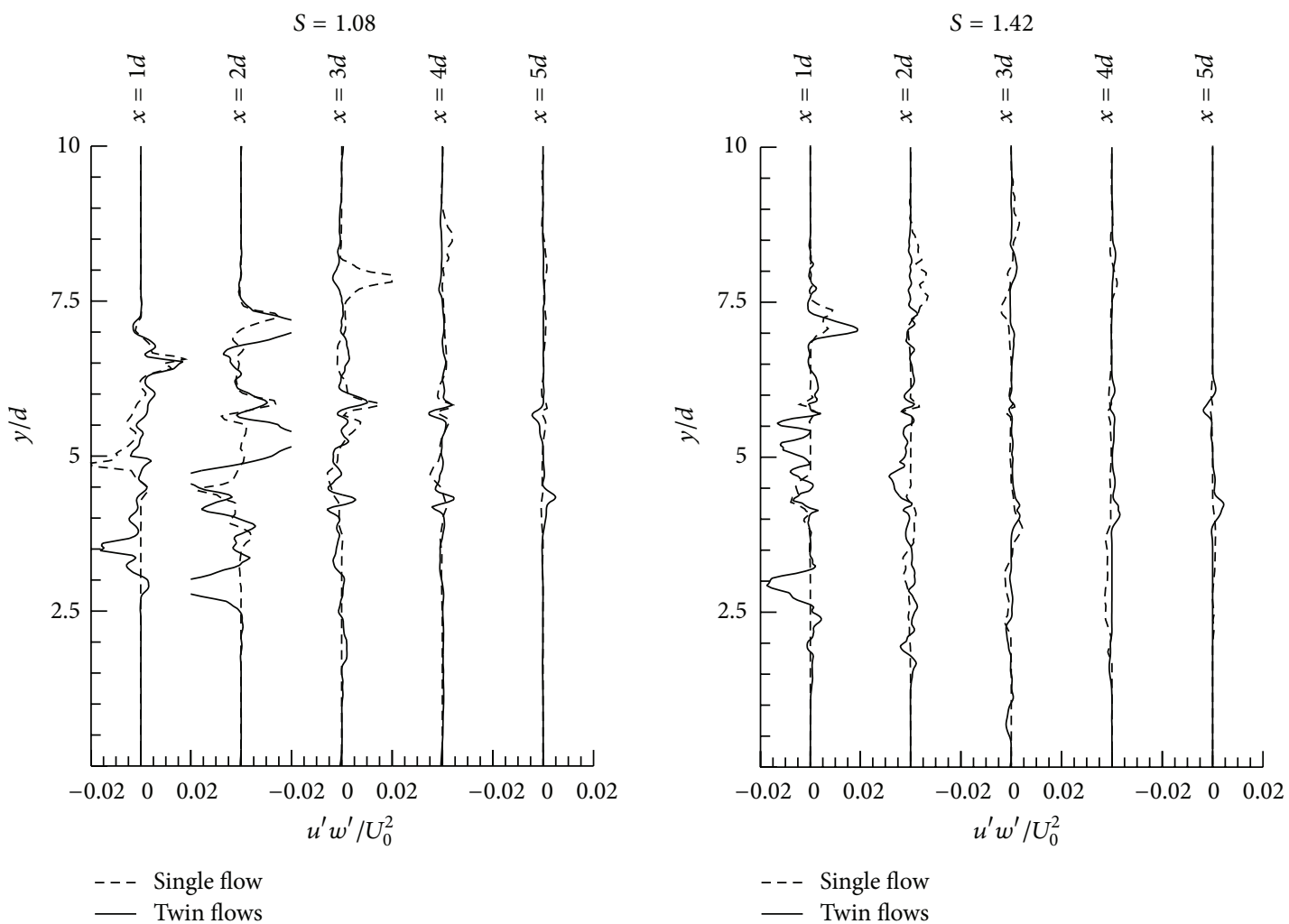

(b)

FIGURE 9: The second order velocity fluctuation profiles of $u^{\prime} w^{\prime}$ for $S=0.68$ (a) and $S=1.08$ and $S=1.42$ (b) in singular and twin swirling flows. 

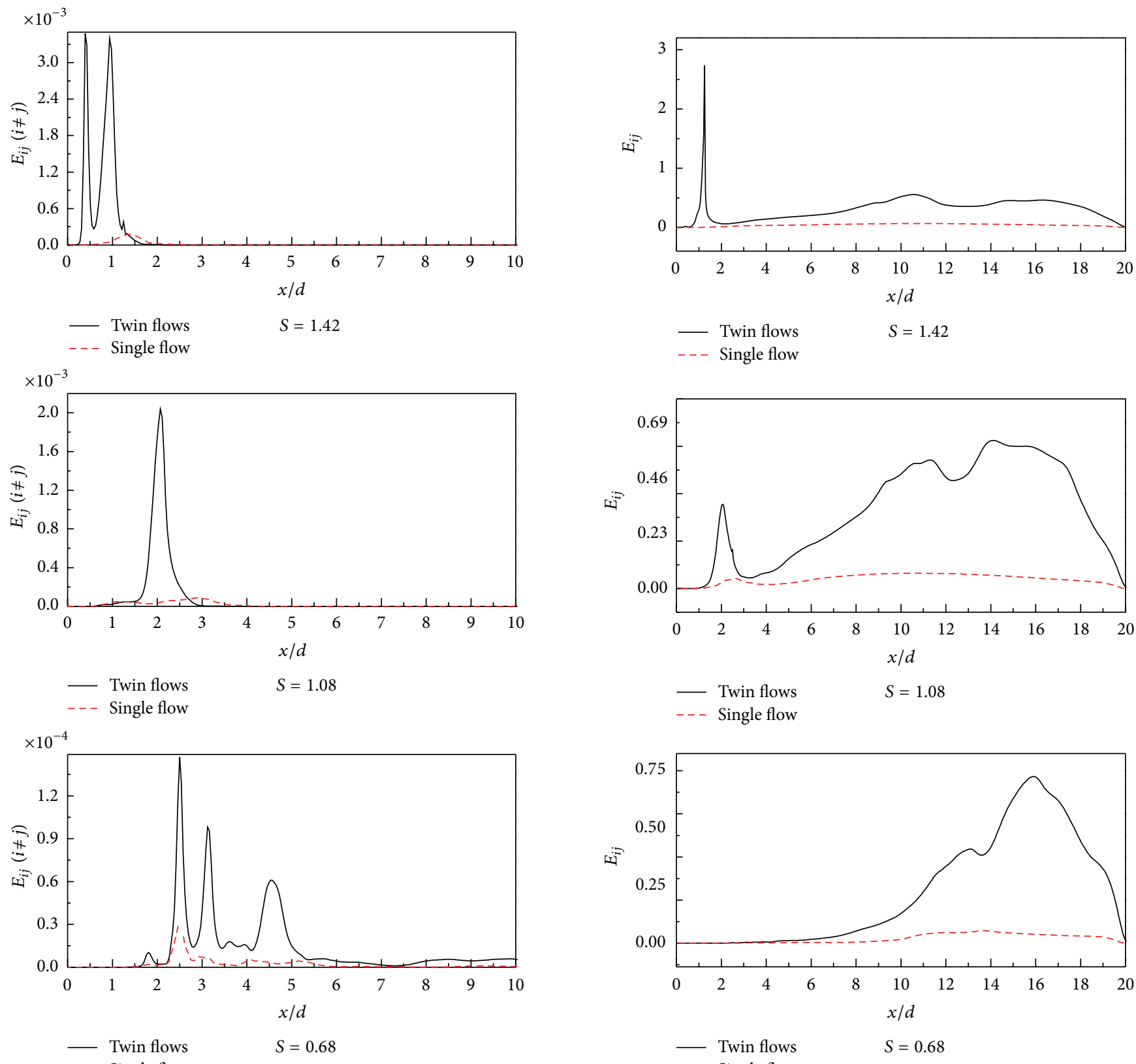

(a)

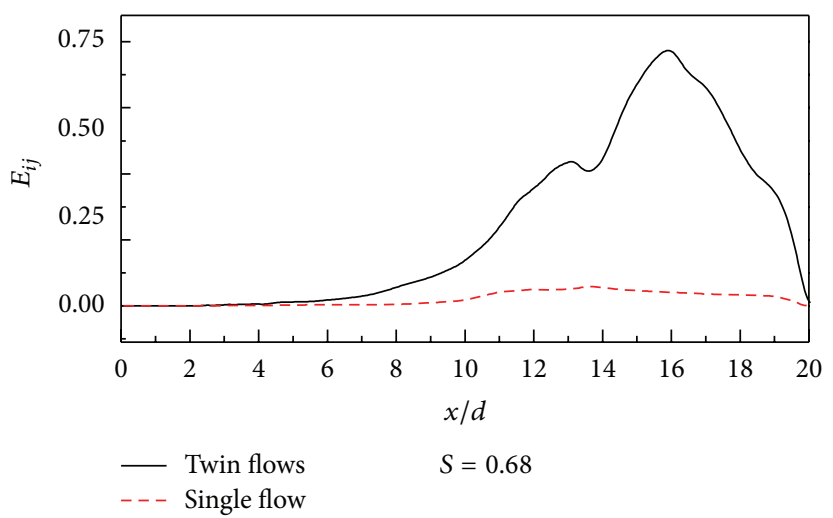

(b)

Figure 10: The $E_{i j}(x)(i \neq j)(\mathrm{a})$ and $E_{i j}(x)(\mathrm{b})$ for different swirl level flows.

Figures 10(a) and 10(b) show $E_{i j}(x)(i \neq j)$ and $E_{i j}(x)$ for different swirl level flows, respectively. No matter what the swirl number is, $E_{i j}(x)$ and $E_{i j}(x)(i \neq j)$, namely, the sum of squares of correlated fluctuations for twin swirling flows, are almost larger than those for single swirling flow. Thus, it can be concluded that the vortex interaction between the twin swirling flows strengthens the correlation of turbulent fluctuations considerably. Moreover, the degree of peak value differences between the twin swirling flows and singular swirling flow increase as the swirling number increases (see the absolute values of $E_{i j}(x)$ and $E_{i j}(x)(i \neq j)$ in Figure 10).
The peak values of $E_{i j}(x)$ and $E_{i j}(x)(i \neq j)$ for both twin swirling flows and single swirling flow increase when the swirling level increases, whereas the peak values for twin swirling flows increase more rapidly.

Moreover, the variation of $E_{i j}(x)(i \neq j)$ (Figure 10(a)) has different characteristics upon the swirl level. For $S=0.68$, the peaks of $E_{i j}(x)(i \neq j)$ mainly occur from $x / d=2.5$ to $x / d=4.5$, with three relatively big peak values. For $S=$ 1.08 , the peaks of $E_{i j}(x)(i \neq j)$ occur at about $x / d=2$, earlier than the former case and with only one peak. For $S=1.42$, the peaks of $E_{i j}(x)(i \neq j)$ occur even earlier at 
about $x / d<1$, with two peaks. The number and location of peaks of $E_{i j}(x)(i \neq j)$ indicate the number and existence of strong vortex-vortex interaction between the twin jets, respectively. More importantly, the peaks occur within a short range in the streamwise direction, which show the localized feature of strong vortex-vortex interaction. Notice that the $E_{i j}(x)(i \neq j)$ mainly indicates the correlation of turbulent fluctuations in different directions. The peaks of $E_{i j}(x)(i \neq j)$ may indicate the existence of strong correlation of turbulent fluctuations in different direction, which could be related to the characteristics of coherent motion and structure of vortices. In other words, the peaks of $E_{i j}(x)(i \neq j)$ show the existence of evident modification of coherent motion of vortex in the twin swirling jets.

In addition, the variation of $E_{i j}(x)$ (Figure 10(b), including variation of $E_{i j}(x)(i \neq j)$ and $E_{i j}(x)(i=j)$, corresponding to the turbulent kinetic energy) shows another aspect upon turbulent kinetic energy modification by the vortexvortex interaction. It is observed that the modification of $E_{i j}(x)$ is no longer localized. The peak values of mediate $(S=$ $1.08)$ and strong $(S=1.42)$ swirl flows occur usually once. After that, a significant continuous augmentation of $E_{i j}(x)$ is always observed, which indicates significant continuous energy transport from the mainstream motion of fluid to the turbulent fluctuation motion. In contrast, for low swirl flows, the initial peak value of $E_{i j}(x)$ disappears, but the continuous augmentation of $E_{i j}(x)$ is also very evident. As aforementioned, the initial peak of $E_{i j}(x)$ shows the main location where strong vortex-vortex interaction happens. Moreover, the strong vortex-vortex interaction may cause a transfer of large-scale vortex into small-scale more complex vortices. Thus, after it, the turbulence is always augmented due to the modification of vortices.

These phenomena show the effect of vortex-vortex interaction on the dissipation and augmentation characteristics of turbulent kinetic energies and validate the influence of correlation of twin swirling flows on modulation of turbulence intensities.

\section{Conclusion}

The present study employed direct numerical simulation to investigate characteristics of twin swirling jets, focusing on the effect of vortex-vortex correlation/interaction on the modification of turbulence characteristics.

The typical vortex-vortex interaction patterns are shown, and distinct differences between twin swirling jets and singular swirling jet are observed and interpreted, especially in the near field. The profiles of normalized velocity fluctuation show the self-similarity characteristics of turbulence in the far field of twin swirling flows.

The second order velocity fluctuations in the twin swirling jets show some fundamental characteristics of turbulence modulation. The localized characteristics of modification of $E_{i j}(x)(i \neq j)$ show the existence of strong vortex-vortex coherent interaction between the jets. The continuous augmentation of $E_{i j}(x)$ shows that the strong vortex-vortex interaction can result in a continuous transport of turbulent kinetic energy from the mainstream to the turbulent fluctuations in the downstream. Thus, basic conclusions can be drawn that the correlation between twin swirling jets strengthens turbulence and Reynolds stress, although they take action differently under the different levels of swirls and have different characteristics of turbulence modification.

\section{Conflict of Interests}

The authors declare that there is no conflict of interests regarding the publication of this paper.

\section{Acknowledgments}

The authors are grateful for the support of this work by the National Natural Science Foundation of China (51106180) and the China Postdoctoral Science Foundation (2013M540964).

\section{References}

[1] M. G. Hall, "Vortex breakdown," Annual Review of Fluid Mechanics, vol. 4, pp. 195-218, 1972.

[2] M. Lessen, P. J. Singh, and F. Paillet, "The stability of a trailing line vortex. Part 1. Inviscid theory," Journal of Fluid Mechanics, vol. 63, no. 4, pp. 753-763, 1974.

[3] G. de Vahl Davis and G. D. Mallinson, "An evaluation of upwind and central difference approximations by a study of recirculating flow," Computers and Fluids, vol. 4, no. 1, pp. 2943, 1976.

[4] O. Lucca-Negro and T. O’Doherty, "Vortex breakdown: a review," Progress in Energy and Combustion Science, vol. 27, no. 4, pp. 431-481, 2001.

[5] A. E. Zohir, "Turbulent heat transfer characteristics and pressure drop in swirling flow at upstream and downstream of an abrupt expansion," Heat and Mass Transfer, vol. 48, no. 3, pp. 529-539, 2012.

[6] L. Y. Xie, Y. Q. Xie, J. Z. Yu, H. X. Gao, and Y. M. Xi, "Effects of rotational acceleration on flow and heat transfer in straight and swirl microchannels," Heat and Mass Transfer, vol. 48, no. 6, pp. 953-964, 2012.

[7] C. O. Iyogun, M. Birouk, and J. A. Kozinski, "Experimental investigation of the effect of fuel nozzle geometry on the stability of a swirling non-premixed methane flame," Fuel, vol. 90, no. 4, pp. 1416-1423, 2011.

[8] A. Adzlan and H. Gotoda, "Experimental investigation of vortex breakdown in a coaxial swirling jet with a density difference," Chemical Engineering Science, vol. 80, pp. 174-181, 2012.

[9] I. V. Litvinov, S. I. Shtork, P. A. Kuibin, S. V. Alekseenko, and K. Hanjalic, "Experimental study and analytical reconstruction of precessing vortex in a tangential swirler," International Journal of Heat and Fluid Flow, vol. 42, pp. 251-264, 2013.

[10] B. A. Younis, B. Weigand, and A. D. Vogler, "Prediction of momentum and scalar transport in turbulent swirling flows with an objective Reynolds-stress transport closure," Heat and Mass Transfer, vol. 45, no. 10, pp. 1271-1283, 2009.

[11] S. Roux, G. Lartigue, T. Poinsot, U. Meier, and C. Bérat, "Studies of mean and unsteady flow in a swirled combustor using experiments, acoustic analysis, and large eddy simulations," Combustion and Flame, vol. 141, no. 1-2, pp. 40-54, 2005. 
[12] S. B. Müller and L. Kleiser, "Large-eddy simulation of vortex breakdown in compressible swirling jet flow," Computers and Fluids, vol. 37, no. 7, pp. 844-856, 2008.

[13] Y. Yang and S. K. Kær, "Large-eddy simulations of the nonreactive flow in the Sydney swirl burner," International Journal of Heat and Fluid Flow, vol. 36, pp. 47-57, 2012.

[14] S. Patil and D. Tafti, "Wall modeled large eddy simulations of complex high Reynolds number flows with synthetic inlet turbulence," International Journal of Heat and Fluid Flow, vol. 33, no. 1, pp. 9-21, 2012.

[15] K. K. J. R. Dinesh, K. W. Jenkins, A. M. Savill, and M. P. Kirkpatrick, "Swirl effects on external intermittency in turbulent jets," International Journal of Heat and Fluid Flow, vol. 33, no. 1, pp. 193-206, 2012.

[16] J. Paik and F. Sotiropoulos, "Numerical simulation of strongly swirling turbulent flows through an abrupt expansion," International Journal of Heat and Fluid Flow, vol. 31, no. 3, pp. 390-400, 2010.

[17] M. Freitag, M. Klein, M. Gregor et al., "Mixing analysis of a swirling recirculating flow using DNS and experimental data," International Journal of Heat and Fluid Flow, vol. 27, no. 4, pp. 636-643, 2006.

[18] M. Freitag and M. Klein, "Direct numerical simulation of a recirculating, swirling flow," Flow, Turbulence and Combustion, vol. 75, no. 1-4, pp. 51-66, 2005.

[19] Z. Q. Yin, J. Z. Lin, K. Zhou, and T. L. Chan, "Numerical simulation of the formation of pollutant nanoparticles in the exhaust twin-jet plume of a moving car," International Journal of Nonlinear Sciences and Numerical Simulation, vol. 8, no. 4, pp. 535-543, 2007.

[20] V. Kolář and E. Savory, "Dominant flow features of twin jets and plumes in crossflow," Journal of Wind Engineering and Industrial Aerodynamics, vol. 95, no. 9-11, pp. 1199-1215, 2007.

[21] Q. Li, G. J. Page, and J. J. McGuirk, "Large-eddy simulation of twin impinging jets in cross-flow," Aeronautical Journal, vol. 111, no. 1117, pp. 195-206, 2007.

[22] D. Fu and Y. Ma, "A high order accurate difference scheme for complex flow fields," Journal of Computational Physics, vol. 134, no. 1, pp. 1-15, 1997.

[23] S. K. Lele, "Compact finite difference schemes with spectral-like resolution," Journal of Computational Physics, vol. 103, no. 1, pp. 16-42, 1992.

[24] A. Jameson and W. Schmidt, "Some recent developments in numerical methods for transonic flows," Computer Methods in Applied Mechanics and Engineering, vol. 51, no. 1-3, pp. 467-493, 1985.

[25] U. Anantha Krishnaiah, R. Manohar, and J. W. Stephenson, "Fourth-order finite difference methods for three-dimensional general linear elliptic problems with variable coefficients," Numerical Methods for Partial Differential Equations, vol. 3, no. 3, pp. 229-240, 1987.

[26] N. Gui, J. Fan, K. Cen, and S. Chen, "A direct numerical simulation study of coherent oscillation effects of swirling flows," Fuel, vol. 89, no. 12, pp. 3926-3933, 2010.

[27] N. Gui, J. R. Fan, and S. Chen, "Numerical study of particleparticle collision in swirling jets: a DEM-DNS coupling simulation," Chemical Engineering Science, vol. 65, no. 10, pp. 3268$3278,2010$.

[28] I. Orlanski, "A simple boundary condition for unbounded hyperbolic flows," Journal of Computational Physics, vol. 21, no. 3, pp. 251-269, 1976.
[29] J. Kim, D. Kim, and H. Choi, "An immersed-boundary finitevolume method for simulations of flow in complex geometries," Journal of Computational Physics, vol. 171, no. 1, pp. 132-150, 2001. 

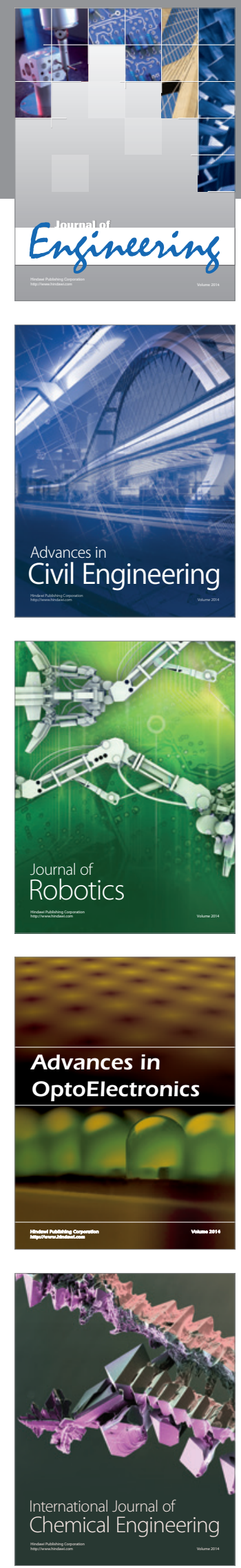

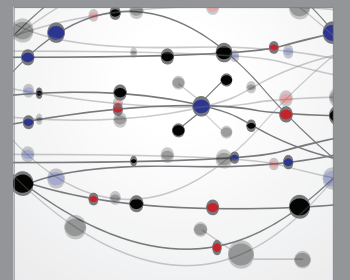

The Scientific World Journal
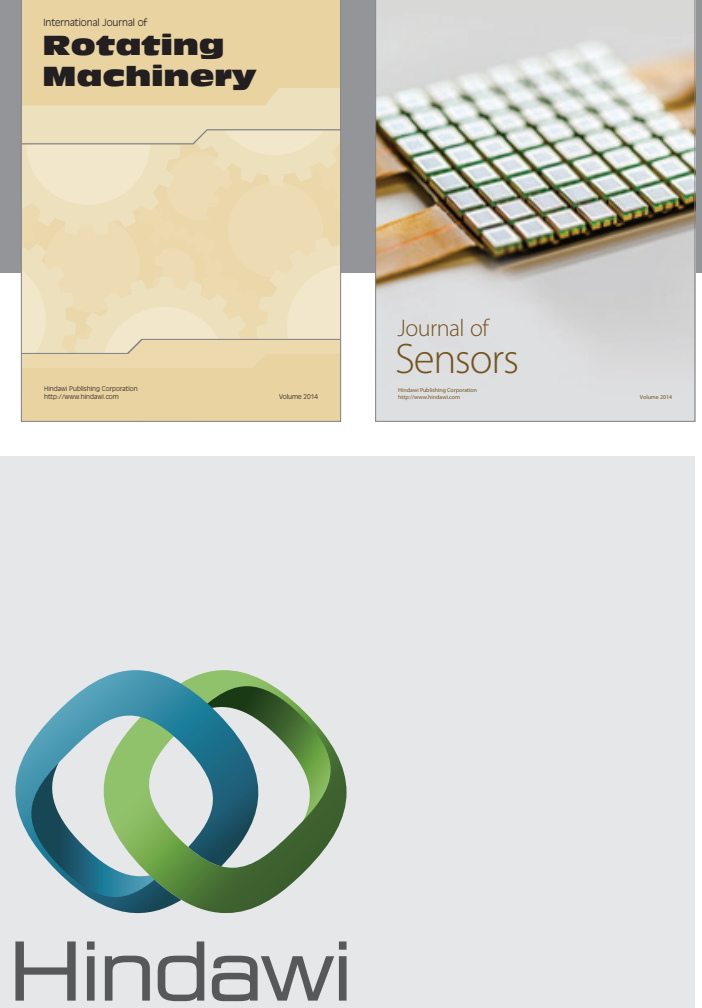

Submit your manuscripts at http://www.hindawi.com
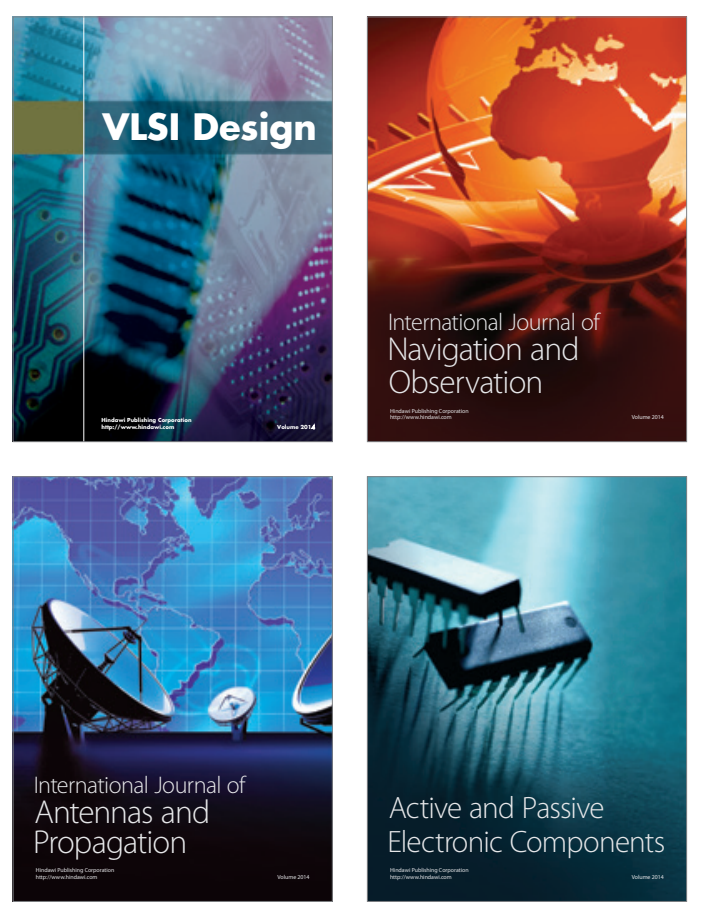
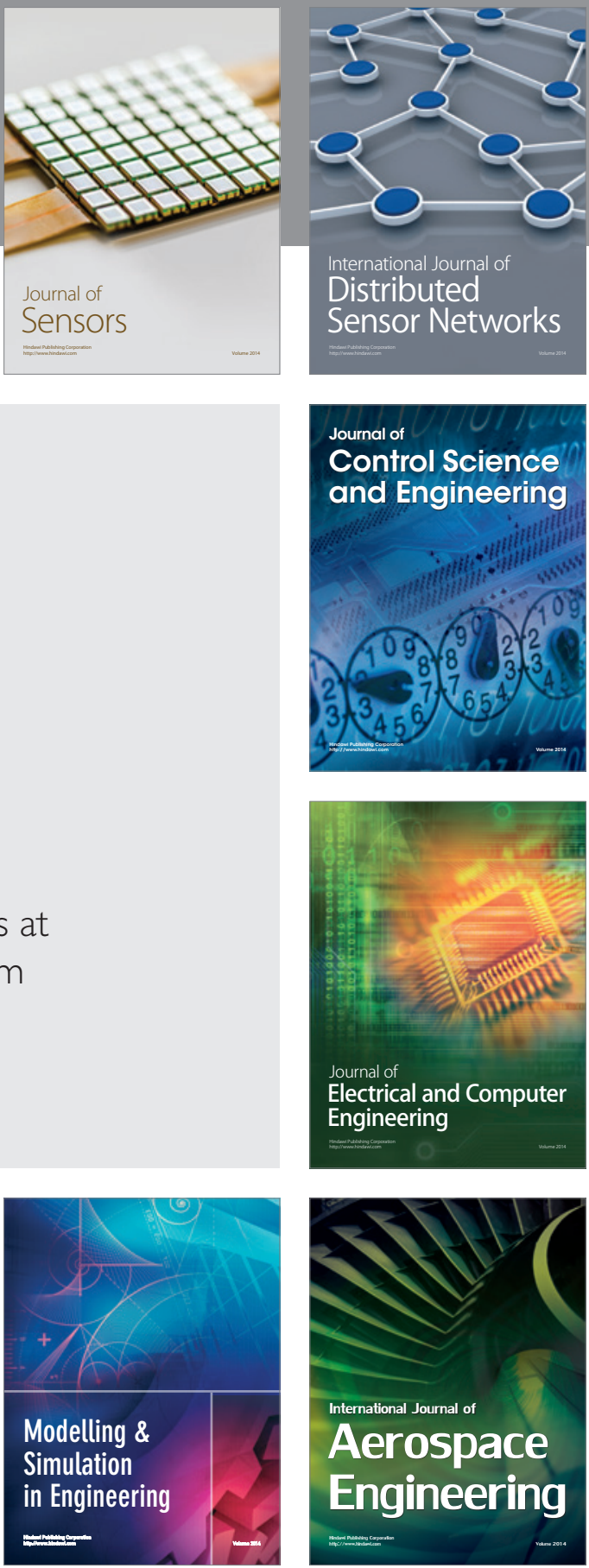

Journal of

Control Science

and Engineering
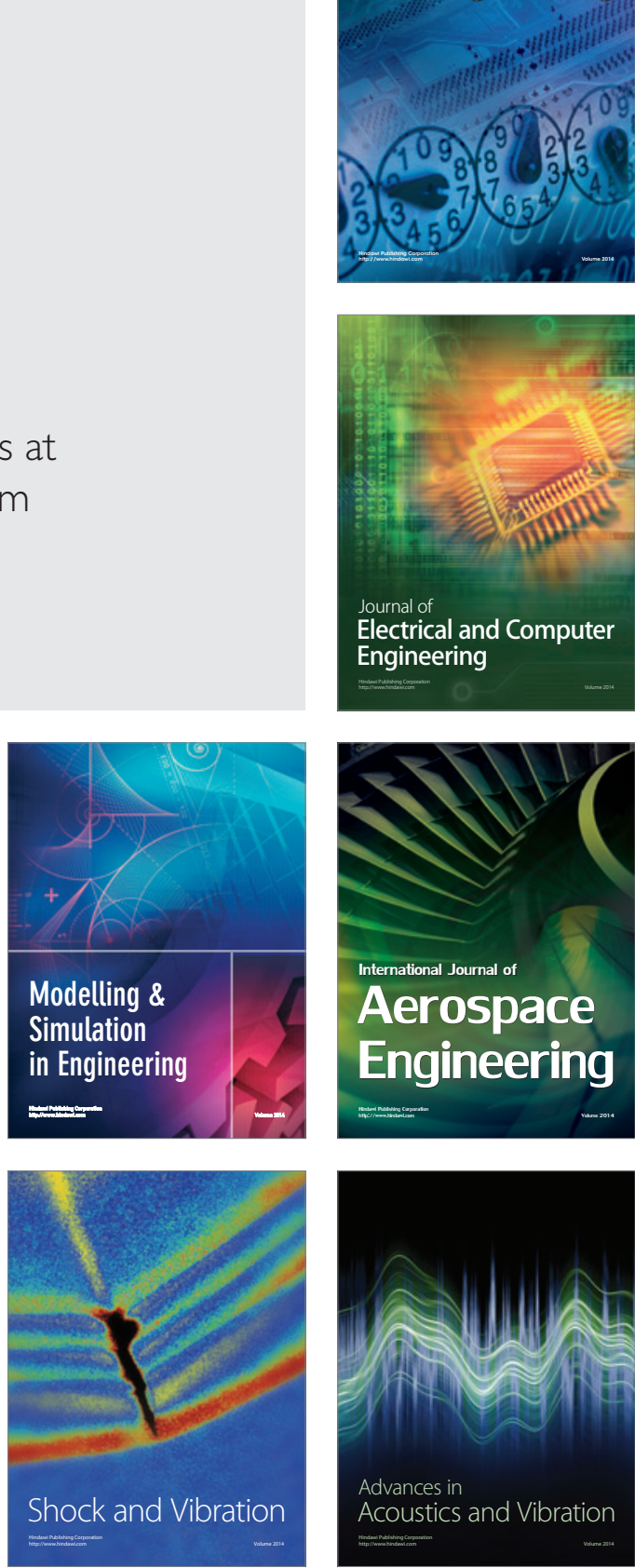